\title{
Disfruta de la experiencia, retrospectiva de una iniciativa de envejecimiento saludable en Andorra (Teruel), 2003-2016
}

\author{
Agustín Quílez Clavero \\ Trabajador SOCIaL \\ Comarca de Andorra-Sierra de Arcos \\ agustinquilez@hotmail.com
}

\section{Resumen:}

Introducción: El programa Disfruta de la Experiencia atesora una larga trayectoria en la organización de actividades de tiempo libre para personas mayores según una filosofía inclusiva, comunitaria y preventiva. Desde 1999 ha organizado más de doscientas actividades para dinamizar a las personas mayores de su municipio.

Materiales y método: El diverso material generado ha servido de base documental para escribir el texto. La metodología es fundamentalmente cualitativa, inspirada en el estudio de casos, ya que se considera que es un caso singular que podría calificarse como buena práctica. 
Resultados: Este programa forma parte de la red de Programa de Promoción de Salud del Gobierno de Aragón. Se destaca su la continuidad a lo largo del tiempo. Esta continuidad se desgrana en varios aspectos: el compromiso por parte del voluntariado, el compromiso de los apoyos tanto ciudadanos, como de las entidades promotoras y por último el compromiso de un equipo profesional. La creación y mantenimiento de un bien relacional durante ese tiempo se considera un logro que redunda en el colectivo de personas mayores que participa en las actividades y en la comunidad.

Discusión de resultados: Las evaluaciones practicadas hasta el momento se han limitado a autoevaluaciones, lo cual se cree que debería ser complementado con una visión externa que ejerza un contraste. Por ese motivo se ha intensificado el esfuerzo de exposición pública del programa que ayude en esta tarea.

Palabras claves: Participación comunitaria, envejecimiento activo, promoción de la salud, trabajo en equipo, medio rural.

\section{Enjoy the Experience, Retrospective of an Initiative for Healthy Aging in Andorra (Teruel), 2003-2016}

\section{Abstract:}

Introduction: The programme Disfruta de la Experiencia (Enjoy the Experience) has accumulated a long history in the organisation of leisure activities for older people according to inclusive, community and preventive thinking. Since 1999 more than two hundred activities have been organised to revitalise the elderly in the municipality.

Materials and method: The diverse nature of the material generated has served as a documentary basis for writing the text. The methodology is fundamentally qualitative, inspired by the case studies, since it is considered to be a unique case that could be defined as good practice.

Results: The programme forms part of the Aragonese Government's Health Promotion Projects network. Attention is drawn to its continuity over the years. This continuity is explored in various aspects: commitment on the part of the volunteers, the committed support 
of both the citizens and the promoters, and finally the commitment of a professional team. The creation and maintenance of a relational product during this time is considered to be an achievement that favours the group of senior citizens participating in activities and in the community itself.

Discussion of results: The reviews carried out so far have been confined to self-evaluation, which it is thought should be complemented by an external viewpoint in a contrastive capacity. For this reason, greater effort has been invested in bringing this programme to the attention of the general public to help in this task.

Key Words: Community participation, active aging, promotion in health, teamwork, rural environment. 



\section{Disfruta de la experiencia, retrospectiva de una}

iniciativa de envejecimiento saludable en Andorra

Agustín Quílez Clavero (Teruel), 2003-2016

\section{INTRODUCCIÓN}

El artículo que se presenta trata de resumir la historia de un programa de envejecimiento saludable organizado según un modelo participativo. En sus dieciséis años de existencia, este programa ha logrado consolidar un trabajo conjunto entre cinco entidades distintas que operan con el mismo sector de población, las personas mayores, en el mismo territorio. La confluencia de esfuerzos ha permitido que un equipo interdisciplinar pueda organizar actividades de envejecimiento activo trascendiendo a los objetivos de su entidad matriz en busca de consolidar actividades alternativas de ocio inclusivas. Con el programa se pretende incidir en los determinantes de salud no biológicos como, por ejemplo, el estilo de vida (Granizo y Gallego, 2007).

La entrega del Premio a las Buenas Prácticas de Trabajo Social, que otorgó el Colegio de Aragón de Trabajo Social, el 4 de noviembre de 2016 (Colegio Profesional de Trabajo Social de Aragón, 2016) estimula para dar a conocer este trabajo que podría ser ejemplo para profesionales que quieran trabajar en una línea parecida. En cierto modo se puede considerar un caso singular que merece ser difundido (Coller, 2005).

El origen del programa se puede fijar en un incidente concreto que posibilitó un momento y un espacio de reflexión conjunta en- 
tre profesionales del ámbito social y educativo (Disfruta de la experiencia, 2016). En otoño de 1998, dos propuestas de programa de envejecimiento activo fueron presentadas a dos departamentos distintos del Ayuntamiento de Andorra, provincia de Teruel. Ambas propuestas presentaban unas características similares. Por una parte, se presentó un programa al Departamento de Cultura y por otra al Departamento de Servicios Sociales. La responsable del Departamento de Servicios Sociales decidió convocar en una reunión a los técnicos ${ }^{1}$ de las áreas para tratar de aunar los esfuerzos que evitaran la duplicidad. En una población de algo más de 8000 habitantes, el esfuerzo conjunto parecía lo más razonable. Esta reunión sirvió para la creación de un equipo motor que de forma permanente velara por la coordinación en la organización de actividades de ocio para personas mayores.

El equipo motor contaba con la participación de técnicos de las cinco entidades siguientes ${ }^{2}$ : Asamblea Comarcal de Cruz Roja; Hogar de personas mayores del Instituto Aragonés de Servicios Sociales ${ }^{3}$; Ayuntamiento de Andorra; Centro de Educación de Adultos y Comarca de Andorra-Sierra de Arcos. Los perfiles profesionales de los técnicos de estas entidades eran y siguen siendo diversas: tres trabajadores sociales, una psicóloga, una terapeuta ocupacional y una profesora de Educación de Adultos. Si bien han cambiado algunas personas, las figuras profesionales han perdurado en el tiempo, así como el compromiso de sus entidades. Se puede afirmar que ha existido un permanente trabajo interdisciplinar e interinstitucional.

Los programas para personas mayores que habían funcionado hasta la fecha de creación de este equipo trataban de dar respuesta a las necesidades de ocio de las personas mayores asignando, un rol pasivo a éstas (Disfruta de la Experiencia, 2016b). El término participación se entendía como la simple asistencia a las actividades organizadas o al consumo de esas actividades. Con el nuevo paradigma que se quería impulsar, se intentó llevar a la práctica, la pretensión de que las personas mayores asumieran, un rol más

${ }^{1}$ Se reconoce el androcentrismo del lenguaje y la alta feminización en profesiones relacionadas con el ámbito socioeducativo, sin embargo, en alguna ocasión, se empleará el genérico masculino por mayor claridad expositiva.

2 Se emplea la denominación actual pero, hay que tener en cuenta desde 1998, los cambios orgánicos sufridos por cada una de las entidades matrices.

${ }^{3}$ En adelante IASS 
activo, según la creencia que tenían mucho que aportar. Partiendo de las experiencias y conocimientos de las personas mayores se podían organizar actividades que promovieran un cambio de actitud. De hecho el lema del folleto informativo (dedicado a la captación de voluntarios) recogía la siguiente frase, basada en un proverbio oriental: Sólo en medio de la actividad desearás vivir 100 años. Recientemente se pronunciaba sobre la labor voluntaria, Montserrat Colomer sobre el tipo de motivación que requiere un voluntario (Colomer, 2016). La mencionada autora declaraba la necesidad de que el voluntario se sintiera a gusto con su labor. Se pretendía pasar de un rol pasivo (consumidor de actividades) a un rol activo (impulsor, promotor de actividades) en busca de alentar su protagonismo.

La situación preexistente, en cuanto al ocio de las personas mayores, presentaba un esquema un tanto repetitivo. Las actividades se basaban en encuentros, con las inevitables celebraciones y en convivencias que solían incluir viajes, comidas y bailes. No era intención del nuevo programa desechar ese tipo de actividades puesto que se valoraron sus virtudes. Por ejemplo, el baile, es un tipo de ejercicio físico recomendado (Ministerio de Sanidad, Servicios Sociales e Igualdad, 2015) y la labor socializadora de los encuentros y convivencias, fomentan una mayor calidad de vida en las personas mayores (Crespo, 2014). Se pensó que las indudables virtudes de esas actividades podrían ser complementadas con otro tipo de actividades, de índole preventiva, inclusiva, solidaria y de promoción de la salud; sin olvidar el componente lúdico, necesario para la motivación del colectivo.

Si bien se pretendía contribuir en el cambio de orientación de las actividades organizadas hasta la fecha, también se tuvo presente hacer frente a la necesidad de envejecimiento activo por estar frente a un fenómeno de evidente importancia, el demográfico. Cuidar la salud de las personas mayores se convierte en todo un reto dadas cifras como la esperanza de vida, que se situaba en los inicios del programa en 78 años (Ministerio de Sanidad, Política Social e Igualdad, 2010) y la tasa de envejecimiento de 16,7 por cien. Es decir se quería intervenir con un colectivo con un peso en la sociedad considerable. 


\section{METODOLOGÍA Y MATERIALES}

Este apartado se estructura en dos partes. Una hablará sobre aspectos organizativos del programa, metodología para la acción y por otra parte se hablará de la metodología de la investigación.

Para el desempeño del trabajo (la organización de las actividades) se contó con inspiración en el método de Investigación-Acción Participativa (Fals, 1993) puesto que se ha dado mucha importancia a la participación comunitaria. Según la metodología de Investigación-Acción Participativa, los ciudadanos deben tomar un papel activo. En el Programa hay una clara diferencia entre los técnicos que gestionan el programa y organizan las actividades y las personas mayores que participan en el mismo. La participación de los mayores se sustancia en el plano operativo (Disfruta de la experiencia, 2016). Las personas mayores que participan en el programa se involucran activamente en el diseño de la actividad puesto que se parte de su propia experiencia, recogida a través de técnicas de recogida de información como cafés tertulias. Sin embargo, la organización global del programa recae en un equipo técnico.

Existen varios materiales de apoyo a la metodología de acción y una organización sistemática de actividades. Todos los ciclos de educación infantil, educación primaria y educación especial, cuentan, al menos con una actividad intergeneracional a lo largo del curso. De este modo se asegura que al menos, en cuatro etapas de su vida escolar, cada niño participe en una actividad de este tipo. Se dispone de materiales como cuadernillos para los docentes y los alumnos en el caso de las charlas. Existen videos que apoyan algunas actividades e incluso se puede hablar de la propia elaboración de videos gracias a la colaboración de los técnicos de la Televisión Local (Disfruta de la experiencia, 2009; 2011)

En lo que respecta a la metodología para la investigación y difusión de resultados, se ha tenido que asumir una labor de evaluación interna. Tal y como recomienda el método de casos (Coller, 2005) se han combinado las técnicas de investigación. Una de esas estrategias ha sido la recopilación de ciertas cifras estadísticas para ganar en objetividad.

Si bien las cifras son importantes, la valoración que los propios participantes hacen de su experiencia (Granizo y Gallego, 2007) 
es el punto clave para considerar la consecución de los objetivos. De esa valoración (de su satisfacción con el programa) también se cuenta con alguna evidencia empírica tanto cualitativa como cuantitativa. Con una metodología basada mayoritariamente en técnicas cualitativas, se da importancia a los discursos que emergen desde los participantes de los programas. Para poder valorar esta variable son precisas observaciones sistemáticas. De este modo se asegura que la recogida de las opiniones de los participantes en los programas se hace de modo organizado, mediante una ficha de evaluación, que se muestra en el anexo I. Por ese motivo se instauró la técnica de la observación participante en prácticamente la totalidad de las actividades organizadas, por parte de los evaluadores (los técnicos del programa), que al mismo tiempo que ayudaban en la organización de esas actividades supervisaban las interacciones que se producían entre los diferentes actores.

Decantarnos por una metodología cualitativa no significa la ausencia de registros. De hecho se han generado buen número de documentos que podrían ser analizados por un evaluador externo para valorar el programa. Ciertos datos objetivos como el número de personas que participan en cada actividad, se han recogido de modo sistemático. Por ejemplo, en el caso de las actividades intergeneracionales, se velaba por recoger cuántos voluntarios (personas mayores) se habían involucrado y cuántos escolares lo habían hecho.

Se ha hablado de la observación participante como técnica para evaluar el programa, con el empleo del registro de una ficha de actividad (Osuna y Márquez, 2000), pero también se puede hablar de otra técnica como es el grupo de discusión. De modo sistemático, el equipo técnico tiene reuniones para organizar las actividades y para evaluar la marcha de las mismas. Estas reuniones son semanales o quincenales, según la carga de trabajo que se soporta. Como se ha apuntado, a estas reuniones, en sentido estricto, no se las podría calificar como grupo de discusión (Ruiz, 1996), sin embargo se cree que hace la función evaluativa de un modo organizado y sistemático, no arbitrario.

En 2014 se empleó la técnica del DAFO para evaluar el programa. En una etapa de madurez del grupo de trabajo, se creyó oportuno analizar lo que ocurría en el programa puesto que la 
primera etapa, (que corresponde con una cierta euforia ${ }^{4}$ ) había quedado atrás.

Con este análisis (primero se pidió una reflexión individual y luego una puesta en común) se querían planificar las estrategias a seguir, teniendo en cuenta el momento y contexto en el que se encontraba el programa, cuyos componentes (personas mayores, comunidad escolar, responsables políticos y equipo técnico) habían experimentado unos inevitables cambios a lo largo del tiempo. En este caso se puede hablar de una técnica de investigación cualitativa que venía a complementar las observaciones sistemáticas. Mediante dinámicas de grupo, se plasmó un análisis participativo del que se extrajeron unas conclusiones y unos aprendizajes para impulsar una evolución en el programa.

Otras fuentes de información que se ha generado y, que se cree conveniente nombrar en este apartado, son las memorias anuales (Disfruta de la experiencia, 2016b), los artículos en prensa (local y comarcal) que se iban realizando, los álbumes de fotografías, los vídeos, los cuadernillos para las actividades escolares, los blogs de los colegios que recogen noticias sobre el programa, y otras fuentes de información que han servido para redactar este texto y que podrían ser empleadas para poder reproducir la experiencia (según una óptica experimentalista) o para investigación evaluativa. Este tipo de registros son aceptados como válidos por la metodología cualitativa (Ruiz, 1996).

Si bien es difícil reproducir exactamente las mismas condiciones en las que se desarrolla el programa (el elemento local es importante), la mayor parte de las mismas pueden ser estudiadas gracias a estos registros. Con esa base se podría experimentar poner en práctica la misma iniciativa en otro ámbito.

\section{RESULTADOS}

Las entidades promotoras de esta iniciativa son cinco: Ayuntamiento de Andorra, Cruz Roja, Comarca de Andorra-Sierra de Arcos, Hogar de Mayores del IASS en Andorra y Centro de Adultos.

${ }^{4}$ De hecho, el programa obtuvo un premio de dos millones de pesetas en 2001 que provocó ese sentimiento. El premio lo entregó la Fundació Agrupació Mutua de Barcelona. 
Las personas que trabajan de manera continuada en este programa son seis: trabajadora social del Hogar de Mayores del IASS, trabajadora social de Cruz Roja, profesora del Centro de Adultos, directora de la Residencia Municipal, terapeuta ocupacional y trabajador social, estos últimos de la Comarca de Andorra Sierra de Arcos. Desde el principio, año 1999, la alianza establecida no ha variado y el compromiso de las entidades nombradas se ha mantenido estable así como el compromiso por parte de los técnicos nombrados. Para presentar este texto se han escogido los últimos 13 años por considerar que se ha seguido una metodología de acción relativamente uniforme.

La continuidad en el tiempo de un conjunto de actividades permite el empleo de la palabra programa (Sánchez, 2007b). Se prefiere esta palabra al término proyecto. Este vocablo alberga un significado diferente puesto que se aplica a un trabajo en ciernes. Según este autor, los programas intergeneracionales deben presentar una serie de características, entre las que se encuentra la continuidad en el tiempo. Las interacciones producidas entre generaciones deben perseguir esa continuidad para lograr su institucionalización.

Según el principio reflejado en el párrafo anterior, el primer resultado que se puede reflejar, es la consolidación del programa y la repetición sistemática de actividades, según una lógica de programación anual.

Constatada esta primera aseveración se hará una aproximación a los efectos resultantes del programa (resultados pretendidos y no pretendidos). Empleando un lenguaje propio de la evaluación de programas, se dará cuenta de los resultados obtenidos, según la teoría del programa pero también de los otros efectos, puesto que han podido darse efectos no esperados o nos buscados (Ligero, 2011).

Teniendo en cuenta que el programa busca la dinamización de las personas mayores, los efectos no buscados intencionalmente, como los derivados de las interacciones con los alumnos de centros escolares (ver gráfico 2), pueden ser calificados como efectos, aunque no son resultados pretendidos inicialmente por el programa.

Estos resultados y esos efectos se logran mediante actividades de diversa índole, aunque destacan, en número las actividades 
intergeneracionales, tal y como se refleja en el gráfico 1. A partir de las experiencias vividas por las personas mayores se organizan charlas y talleres. Se describen algunos ejemplos de estas actividades. Un ejemplo lo constituye la actividad sobre los juegos tradicionales. Las personas mayores hablan de los juegos de su infancia en el colegio y unos días más tarde, un grupo de personas mayores, van al colegio a jugar con los niños. Otros ejemplos de actividades organizadas son los cursos de educación para la salud (formativas en el gráfico 1) para mayores o las visitas a exposiciones para personas con movilidad reducida (para lo cual se cuenta con transporte adaptado). Esta última actividad se dirige principalmente a personas de edad más avanzada y con más dificultades. En el gráfico 1 se representa como de integración.

El programa cuenta con un sistema de evaluación de acorde con el volumen de actividad y presupuesto que supone. Una ficha de actividad que registra el desarrollo de las actividades es una de las herramientas principales y la que permite la confección de las estadísticas, (ver gráficos 1 y 2; y tabla 1).

Sin embargo, teniendo en cuenta que se organizan aproximadamente veinte actividades anuales y se han movilizan alrededor de 110 personas mayores, la orientación de las técnicas empleadas es cualitativa.

\section{Gráfico 1: № de actividades realizadas en los últimos 13 años}

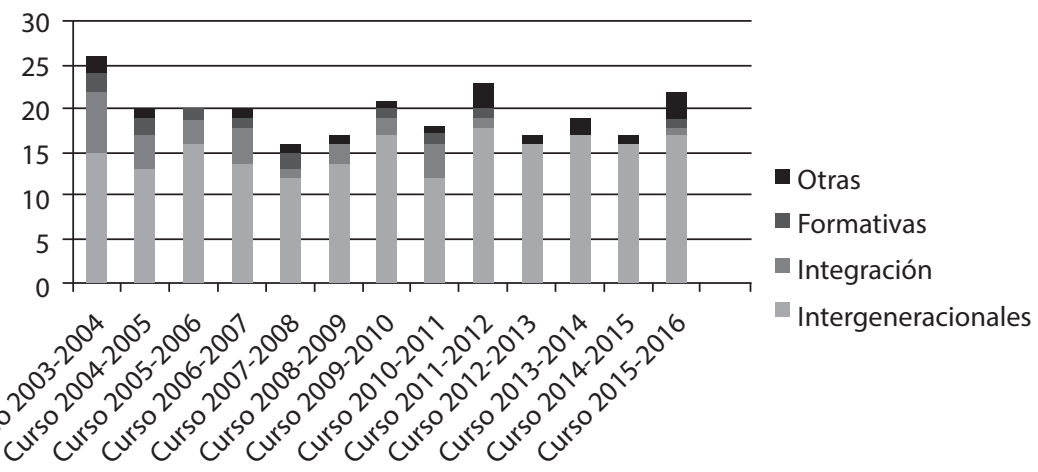

Fuente: Elaboración propia a partir de memorias anuales del Programa 
La memoria anual (Disfruta de la experiencia, 2016b), que suele ser un resumen de las actividades realizadas, de acuerdo con García (2012), no es en sí misma la herramienta idónea para la evaluación en un sentido estricto. Sin embargo recoge una serie de datos que reflejan el desempeño del programa. Si bien su mayor utilidad puede hallarse en la rendición de cuentas, aporta información que, al menos, en parte ayuda a desentrañar, cómo se desarrolla este programa de envejecimiento saludable. Sí que se considera que la observación participante es una técnica clave de investigación que serviría más específicamente para esos fines (ver tabla 1). Con la observación directa se puede comprobar de un modo más fidedigno si los objetivos derivados de la teoría del programa se van cumpliendo. El análisis debe responder principalmente a una cuestión: verificar si las actividades han logrado dinamizar el tiempo libre de las personas mayores. Existe otra pregunta implícita que es saber cómo se han sentido las personas mayores en la actividad. Para ello se emplea una hoja de registro (ver tabla en anexo 1) que no solo verifica si la actividad se ha realizado. También se valora la sensación que (a juicio del observador) ha tenido la persona mayor (o grupo de personas mayores) al realizar la actividad.

Por lo expuesto se deduce que no sólo hay un interés por contabilizar el número de actividades y el número de participantes (ver gráficos 1 y 2). Existe un interés por las sensaciones de los participantes que puedan asegurar un cumplimiento pleno del objetivo y, como consecuencia, la permanencia de ese participante en el programa en futuros ejercicios, es decir su identificación y fidelización con el programa. 
Gráfico 2: Nº participantes últimos 13 años

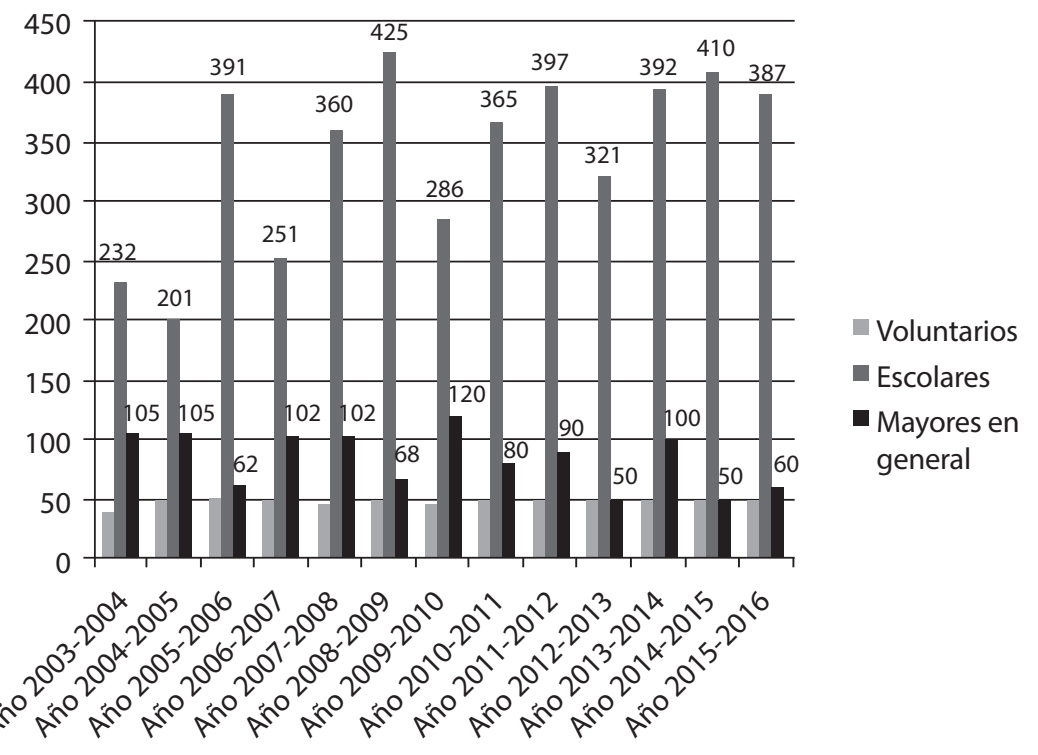

Fuente: Elaboración propia a partir de memorias anuales del Programa

Es importante señalar la importancia del transporte adaptado para promover la participación de personas con problemas de movilidad (Disfruta de la experiencia, 2016). De este modo se responde al objetivo inclusivo. Hay que tener en cuenta que existen profesionales en el equipo técnico cuya formación (psicóloga experta en Gerontología y terapeuta ocupacional) permite que se incluyan a personas con limitaciones funcionales en ciertas actividades, tras estudio individualizado de cada caso.

Como un efecto del programa, se puede apuntar la gran cantidad de escolares que han participado en actividades de intercambio generacional a lo largo del tiempo. La cifra va oscilando, año tras año. Se puede señalar que en once periodos el número de escolares partícipes de las actividades ha superado la cifra de 250. 
Figura 1. Alianzas estables del Programa.

Otras alianzas, según actividades
Asociaciones de

mayores (3)

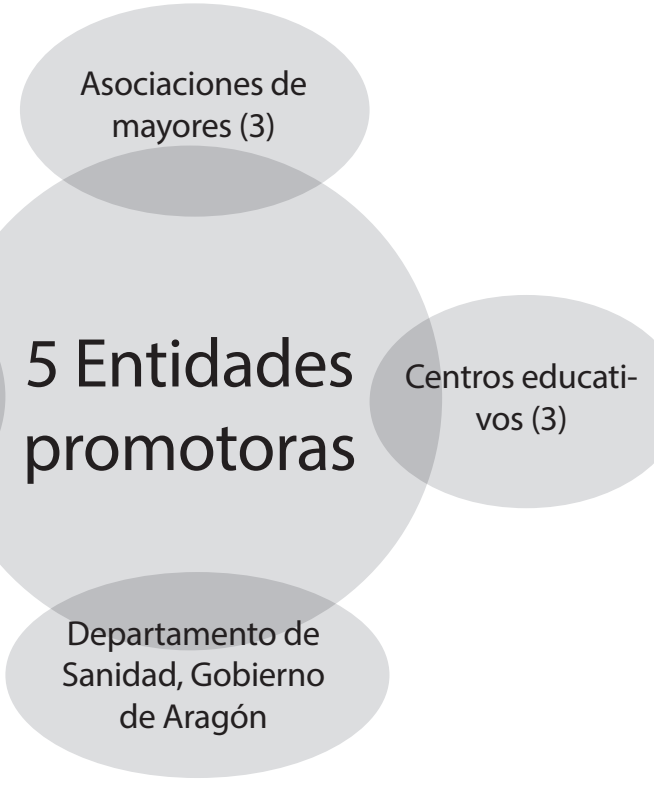

Fuente: Elaboración propia

Este programa se ha configurado, desde sus inicios, como una coordinadora de actividades de ocio saludable para las personas mayores que ha precisado de continuas alianzas (ver figura 1). Según la bibliografía consultada, los programas también son evaluados según la red de alianzas que atesora (Blasco y Subirats, 2009), lo cual se verifica con los datos aportados. Para posibilitar el programa ha sido preciso el concurso de cinco entidades promotoras además de contar con otras alianzas como las asociaciones de mayores o los centros educativos. Dos colegios de primaria, el Instituto de Secundaria y un colegio de Educación Especial están involucrados en esta iniciativa. También ha sido precisa la movilización de voluntariado y la implicación de 3 asociaciones de mayores. El programa pertenece desde 2003 a la Red de programas de promoción de salud del Departamento de Salud del Gobierno de Aragón. Esta entidad contribuye con jornadas de intercambio, con formación conjunta y también con financiación vía subvenciones. Anualmente se celebran jornadas de intercambio de programas en 
la cual se da un aprendizaje mutuo. También se dan otras alianzas puntuales según el tipo de actividad que se organiza. En el gráfico 3 se puede observar cómo la interacción entre entidades es una constante. También es importante señalar que estas alianzas tienen sus reglas pero no están reguladas en ningún documento, simplemente perduran en el tiempo.

\section{Gráfico 3: Entidades involucradas}

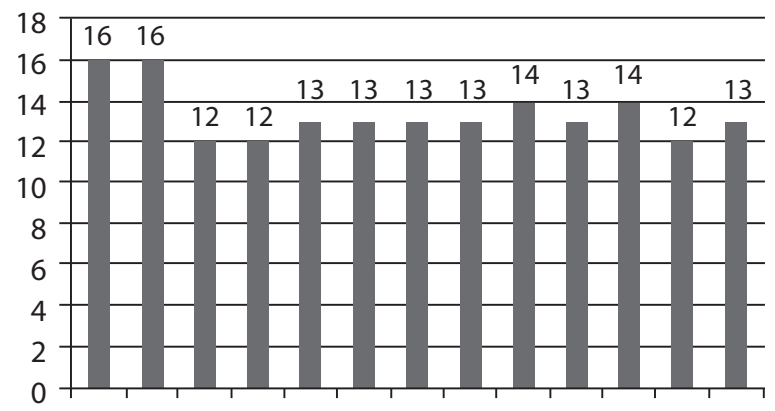

Entidades involucradas

Fuente: Elaboración a partir de datos de Disfruta de la experiencia (2016b)

\section{DISCUSIÓN DE RESULTADOS}

En los resultados se constata la continuidad de un programa que promueve no sólo actividades sino también una importante red de alianzas a lo largo del tiempo, lo que se puede relacionar con un trabajo comunitario y en red.

Los resultados concretos muestran continuidad en un grupo de personas mayores voluntarias que participa con un rol participativo. Alrededor de 50 personas al año se involucran en las actividades. Los efectos del programa no se limitan a la dinamización de las personas mayores sino que también repercute en la Comunidad Escolar. Los centros educativos asumen en su programación anual que cada año se participará en el programa. 
Si bien los efectos del programa son conocidos desde el punto de vista de ese grupo de aproximadamente 50 personas mayores, no se puede decir lo mismo de los efectos que se provocan en el medio escolar. Se tiene la creencia, que se redunda en el apoyo de ciertas asignaturas. También se tiene la creencia de que se fomentan relaciones espontáneas entre personas mayores y niños, más allá del aula, pero no se puede constatar esa hipótesis, desde un punto de vista estadístico.

En repetidas ocasiones las memorias hablan de la consecución de los objetivos. Ciertamente las cifras expuestas, constatan una dinamización de las personas mayores. Los materiales producidos, como cuadernillos para los colegios, los vídeos elaborados o los álbumes de fotografías dan testimonio del logro de ese objetivo central. Así mismo las fichas de actividad corroboran este cumplimiento de objetivos.

Sin embargo, también se manifiesta la necesidad de ahondar en cuestiones de metodología. (Disfruta de la experiencia, 2016b). En particular se aboga por adoptar algún tipo de evaluación más sistemática. En este apartado se puede aducir que cada año se pide presupuesto a las entidades promotoras lo cual, en cierto modo es una suerte de evaluación. Los responsables de la toma de decisiones, gracias a la información de que disponen, adoptan la decisión de que el programa continúe. Por este motivo, de sometimiento del programa a la voluntad anual de los responsables públicos, se puede interpretar que se enjuicia anualmente el trabajo realizado.

Someter el programa a las periódicas interacciones de la Red de Proyectos de Promoción de Salud da también una garantía sobre la profesionalidad de la labor realizada. Se somete a exposición pública el contenido de los programas y también se somete la profesionalidad de los ejecutores que, de alguna manera, rinden cuentas de lo realizado periódicamente. También se considera que haber recibido un premio de un colegio profesional es garantía de calidad del trabajo realizado.

Se podrían poner en práctica otras actividades, se podría innovar en la metodología y se podía aplicar algún tipo de evaluación externa. Sin embargo, debido a las dimensiones del programa hay que pensar que esos hipotéticos nuevos retos deben adecuarse a esas dimensiones. 
El hecho de presentar un programa innovador tiene sus ventajas e inconvenientes. Lo nuevo puede seducir pero al mismo tiempo puede provocar desconfianza. Esa desconfianza debe contrarrestarse con una presentación pública de calidad en todos los ámbitos posibles y con diferentes registros según cada ámbito. Mariano Sánchez para valorar los programas intergeneracionales (2007b:10) argumentaba lo siguiente:

Sólo si contamos con evidencias bien fundamentadas de los beneficios que aportan a niños, jóvenes, adultos y mayores, ya se trate de personas dependientes o no, estas prácticas conseguirán ganarse el respeto, la confianza y el reconocimiento que merecen.

La garantía de un buen funcionamiento de un programa es someterlo a evaluación. "Lo que no se puede medir no se puede evaluar. Lo que no se puede evaluar no se puede mejorar" (Diputación de Barcelona, 2016). Tras el largo recorrido del programa no sólo se busca un reconocimiento profesional. Distinguían Stufflebeam\&Shinkfield (1993), los programas que tenían mérito de aquellos que respondían a una necesidad y se postulaban para dar respuesta a la misma. En ese punto se quiere poner al programa.

Pese a haber pasado por varios filtros evaluativos, el programa debe seguir sometiéndose a unos mecanismos de evaluación y de transparencia que continúen su exposición pública (profesional y no profesional). De este modo se facilitaría la reconstrucción (si fuera precisa) de sus métodos de trabajo. La presencia en redes profesionales, en Jornadas y Congresos, el empleo de Internet y la difusión de las actividades pueden ser oportunidades para lograr mayor reconocimiento (o por el contrario crítica y oportunidad de mejora).

\section{BIBLIOGRAFÍA}

Blasco, J. y Subirats, J. (2009). Consideraciones generales sobre la evaluación de políticas públicas. Las especificidades de las políticas sociales. Documentación Social, $n^{\circ} 154,39-58$.

Colegio Profesional de Trabajo Social de Aragón (2016). Resonancias del V Acto del Trabajo Social en Aragón. Obtenido el 13 de noviembre de 2016 de: http://www.trabajosocialaragon.es/ 
actualidad/2016/resonancias-del-v-acto-del-trabajo-socialen-aragon-valor-reconocimiento-y-compromiso-2016-teruel4-noviembre.php

Coller, X. (2005). Estudio de casos. Madrid: Centro de Investigaciones Sociológicas.

Colomer, Marta (2016). Voluntariado en los Servicios Sociales de proximidad. XIII Congreso de la Asociación estatal de directores y gerentes de servicios sociales. Fuenlabrada (Madrid)

Crespo, M. A. (2014). Participació social de les persones majors. Viure en Salud, 99, 17-19.

Diputación de Barcelona. (2016). Círculos de Comparación. Jornadas dedicadas a los Servicios Sociales. Material no editado. Barcelona

Disfruta de la experiencia (2004). Programa de dinamización de personas mayores. Memoria para Premio Infanta Cristina. Material no editado. Andorra (Teruel)

Disfruta de la experiencia (2009). Diez años disfrutando. Material no editado. Andorra (Teruel)

Disfruta de la experiencia (2011). Elaboración de jabón casero. Televisión Local de Andorra: Andorra (Teruel)

Disfruta de la experiencia (2016). Informe para Colegio de Trabajo Social de Aragón. Material no editado. Andorra (Teruel)

Disfruta de la experiencia, (2016b). Memoria de actividades 20032016. Material no editado. Andorra (Teruel)

Fals, O. (1993). La investigación participativa y la intervención social. Documentación Social, 92,9-21.

García, G.A. (2011). Sistemas de información y evaluación de programas sociales. En Esther Raya Díez (coord.) Herramientas para el diseño de programas sociales. Universidad de la Rioja. Logroño

Granizo, C. y Gallego, J. (coords). (2007). Criterios de calidad de la Red Aragonesa de de promoción de salud. Zaragoza: Gobierno de Aragón.

Ligero, J.A. (2011). Dos métodos de evaluar: criterios y teoría del programa. Madrid: CEU.

Ministerio de Sanidad, Política Social e Igualdad (2010). Esperanzas de vida en salud en España 1986-2007. Obtenido el 1 de 
septiembre de 2016 de: http://www.msssi.gob.es/estadEstudios/estadisticas/inforRecopilaciones/docs/ESPERANZAS DE VIDA SALUDABLES EN ESPANA ESPANOL-2010.pdf

Osuna, J.L. y Márquez, C. (2000). Guía para la evaluación de las políticas públicas. Sevilla: Instituto de Desarrollo Regional.

Ruiz, J. I. (1996). Metodología de la investigación cualitativa. Bilbao: Universidad de Deusto.

Sánchez, M (dir) (2007). Programas intergeneracionales: Hacia una sociedad para todas las edades. Barcelona: La Caixa. (2007b). La evaluación de los programas intergeneracionales. Madrid: IMSERSO.

Sánchez, M., Díaz, P., López, J., Pinazo, S. y Sáez, J. (2008). Descripción, análisis y evaluación de los programas intergeneracionales en España. Modelos y buenas prácticas. Resumen ejecutivo. Obtenido el 25 de agosto de 2016 de: http://www. imserso.es/InterPresent1/groups/imserso/documents/binario/ idi172 06ugranada.pdf

Sánchez, M., Kaplan, M. y Sáez, J. (2010). Programas intergeneracionales, guía introductoria. Madrid: IMSERSO.

Shanon, S. (2012). Developing Communities for all agesInternational: Best Practice on Intergenerational Programmes. Obtenido el 25 de agosto de 2016 de: http://agefriendlyireland.ie/ wpcontent/uploads/2014/06/Developing_Communities_for all Ages.pdf

Stufflebeam, D. L. y Shinkfield, A. J. (1993). Evaluación sistemática. Guía teórica y práctica. Madrid: Paidós. 
ANEXO 1: Tabla 1: Hoja de registro de actividades del programa

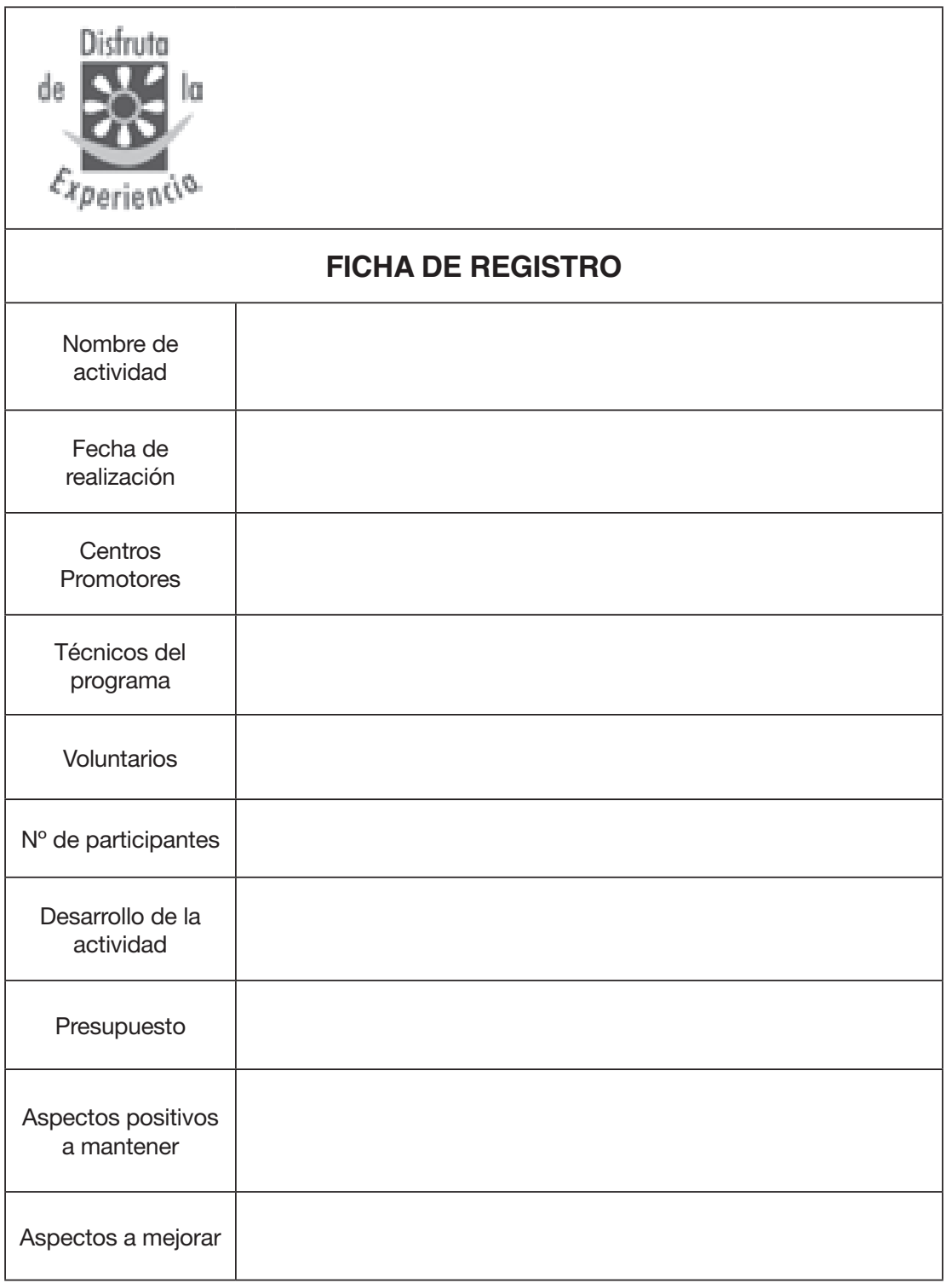

Fuente de Información: Elaboración propia a partir de Disfruta de la experiencia (2016b) 